\title{
Atypical acute retinal necrosis accompanied 1 casamatax by Terson's syndrome: a case report
}

\author{
Jong Young Lee ${ }^{1}$, Dong Yoon Kim², Hye Jin Lee', Jin Ho Jeong ${ }^{1}$, Sung Pyo Park ${ }^{3}$ and Jin Young Kim ${ }^{*}$ (D)
}

\begin{abstract}
Background: Acute retinal necrosis (ARN) has characterized by panuveitis, vitritis, severe vaso-occlusive vasculitis, and diffuse necrotizing retinitis. There are no case reports on atypical ARN combined with Terson's syndrome. Herein, we report a case of ARN with atypical clinical features combined with Terson's syndrome that we successfully treated by intravitreal ganciclovir injection.

Case presentation: A 64-year-old man visited our eye clinic with a complaint of decreased visual acuity in his right eye. At the initial visit, his best corrected visual acuity was 20/125 in the right eye. Slit-lamp examination demonstrated mild hyperemia, keratic precipitates, and anterior chamber inflammatory reaction. Fundus examination revealed multiple diffuse white-yellowish infiltrations in the peripheral retina combined with dot hemorrhages. Ultra-wide-field fluorescence angiography showed obstructive arteritis with peripheral non-perfusion and leakage from the retinal vessels. As a result of the PCR analysis, varicella zoster virus DNA was identified in the aqueous humor. Under the diagnosis with VZV-mediated ARN, we started with intravenous acyclovir and oral prednisolone. After 3 days of the above treatment, the anterior chamber inflammation and vitreous opacity were increased. On fundus examination, multiple whitish infiltrations were increased. In addition, newly developed vitreous and peripapillary hemorrhages were detected. On the T2 brain magnetic resonance imaging (MRI) demonstrated a sub-acute or old hemorrhagic infarction in the right occipital lobe, and contrast-enhancing lesions in the right basal ganglia. The spinal tapping was performed in the department of neurology in our hospital at the time when the patient complained of headache, and intracranial pressure was $31 \mathrm{mmHg}$. Under the diagnosis of ARN with Terson's syndrome, we started intravitreal ganciclovir $(2 \mathrm{mg} / 0.5 \mathrm{ml})$ injections. After 5 intravitreal ganciclovir injections over a period of 8 months, the diffuse whitish infiltrating retinal lesions combined with dot hemorrhage were decreased. The vitreous and peripapillary hemorrhage was significantly reduced. There was no recurrence in the patient's right eye, in which his visual acuity had improved to 20/60.
\end{abstract}

Conclusions: In the event of a poor response to traditional treatment such as intravenous acyclovir, intravitreal ganciclovir may have a role as an adjunctive therapy in patients of VZV associated ARN combined with Terson's syndrome.

Keywords: Acute retinal necrosis, Intravitreal ganciclovir injection, Terson's syndrome

\section{Background}

Acute retinal necrosis (ARN) was first described in 1971 in a Japanese journal as being characterized by panuveitis, vitritis, severe vaso-occlusive vasculitis, and diffuse necrotizing retinitis. ARN preferentially affects the peripheral retina $[1,2]$. The condition usually presents unilaterally, but bilateral cases have been described [3]. Most reported

\footnotetext{
* Correspondence: muse1016@naver.com

'Department of Ophthalmology, Jeju National University Hospital, Jeju National University School of Medicine, 1753-3 Ara-1 Dong, Jeju-Si, Jeju Self-Governing Province, Republic of Korea

Full list of author information is available at the end of the article
}

cases have been caused by varicella zoster virus (VZV), which accounts for $50 \%-80 \%$ of cases. However, herpes simplex virus (HSV) type 1 or 2 has also been the presumptive causative agent in some cases [4-6].

The diagnosis of ARN is usually based on clinical appearance and confirmation of causative viral DNA using serum or intraocular fluid antibody testing, viral culture, retinal biopsy, and polymerase chain reaction (PCR) $[7,8]$. It is now more preferable to test focal samples rather than conducting a serum antibody titer for viruses. Use of polymerase chain reaction (PCR) system in aqueous humor and vitreous fluid 
samples is useful for identifying the viral origin. PCR is currently the preferred method of viral diagnosis [9-11].

The initial treatment for ARN is intravenous acyclovir (1500 $\mathrm{mg} / \mathrm{m}^{2} /$ day) for $5-10$ days, followed by oral acyclovir (800 mg five times/day) for 4-6 weeks. Intravitreal foscanet or ganciclovir injection may also have a role as adjunctive therapy in the management of patients with ARN. Intravitreal foscarnet in combination with systemic antiviral therapy may reduce the risk of retinal detachment in VZV associated ARN [12, 13].

In this report, we describe an unusual case of ARN with atypical clinical features accompanied by Terson's syndrome that was successfully treated by intravitreal ganciclovir injection.

\section{Case presentation}

A 64-year-old man visited our eye clinic with a two-day history of decreased visual acuity in his right eye. The patient presented with a medical history of hypertension and diabetes mellitus. He stated that his vision had gradually worsened in recent days.

At the initial visit, his best-corrected visual acuity was $20 / 125$ in the right eye and 20/25 in the left eye, and intraocular pressures were $18 \mathrm{mmHg}$ and $13 \mathrm{mmHg}$, respectively. Slit-lamp examination demonstrated mild hyperemia and keratic precipitates. The eye also showed inflammation in the anterior chamber and anterior vitreous, which was scored as grade $2+$ and grade $1+$ according to the Standardization of Uveitis Nomenclature Working Group guidelines [14]. Fundus examination of the right eye showed multiple and diffuse whiteyellowish infiltrations combined with retinal dot hemorrhages in the peripheral retina (Fig. 1). The left eye was completely normal. Ultra-wide-field fluorescence

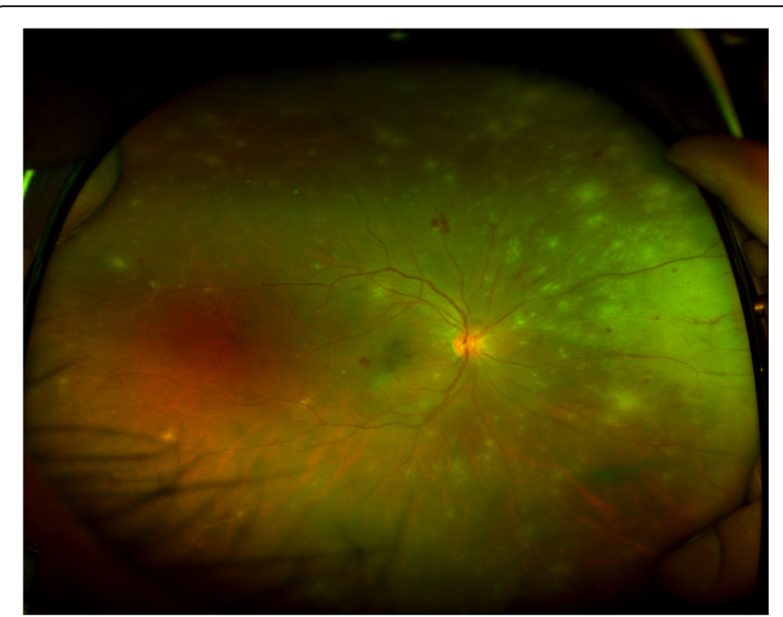

Fig. 1 Ultra-wide-field photograph of the right eye at the initial visit. The mild vitritis with vasculitis and confluent retinitis in the nasal periphery are noted

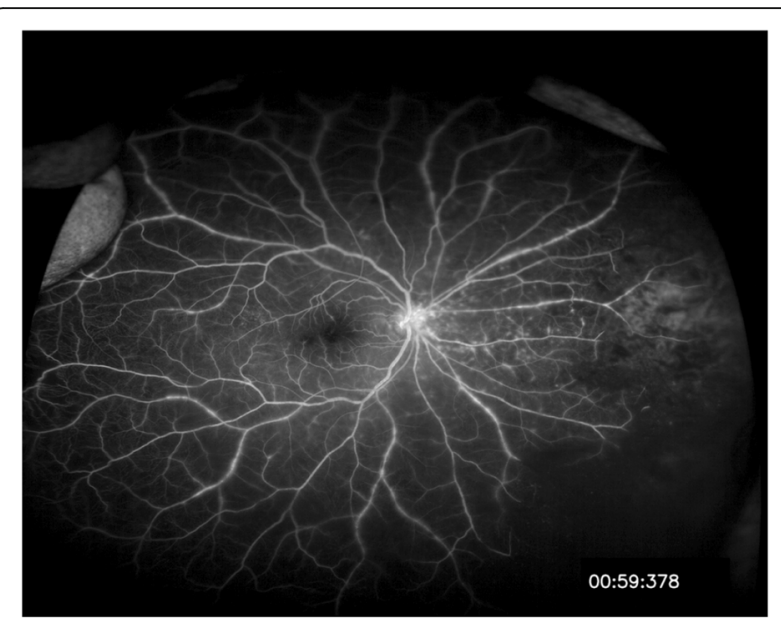

Fig. 2 Ultra-wide-field fluorescence angiography showed diffuse arteriolar and venular occlusions at the initial visit. Hyperfluorescence is prominent in the nasal area

angiography showed obstructive retinal arteritis with peripheral non-perfusion and late leakage from the retinal vessels in the right eye (Fig. 2).

The patient was hospitalized for further evaluation and appropriate management. Basic laboratory test and serology study were performed. The serological tests were positive for HSV type 1 immunoglobulin G (IgG), VZV IgG, Epstein-Barr virus viral capsid antigen IgG, and Toxoplasma-specific IgG antibodies. Anterior chamber paracentesis and PCR analysis of the aqueous humor have been performed. VZV DNA was identified in the aqueous humor. But, DNA for HSV-1, HSV-2, and cytomegalovirus was not detected. Given the impression of VZV associated ARN, treatment was started with intravenous acyclovir (1200 $\mathrm{mg}$ three times a day). Oral

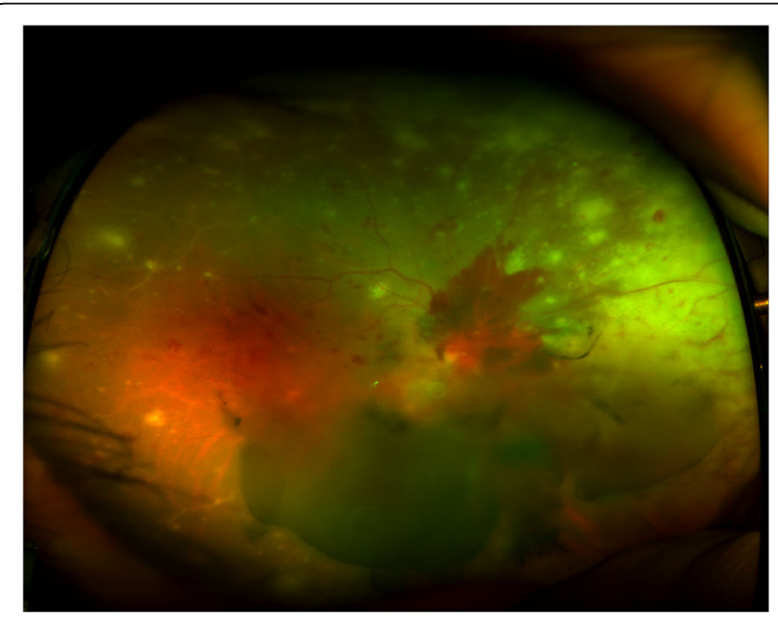

Fig. 3 Ultra-wide-field photograph of the right eye at 3 days after admission. One of the whitish lesions was enlarged and had progressed to the posterior pole, accompanied by vitreous and peripapillary hemorrhage 

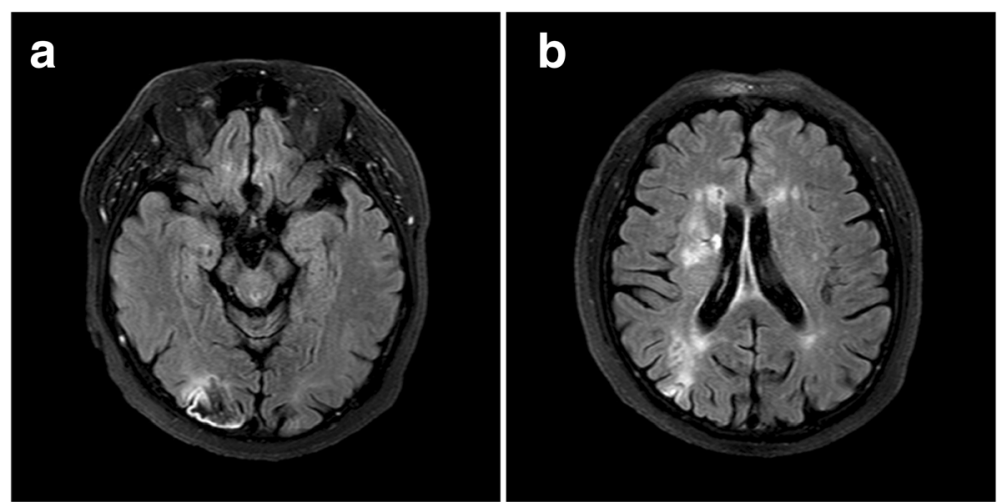

Fig. 4 T2-weighted magnetic resonance images $(\mathbf{a}, \mathbf{b})$ of the brain. a A sub-acute or old hemorrhagic infarction in the right occipital lobe. $\mathbf{b}$ Contrast-enhancing lesions in the right basal ganglia that may be a cause of the suspected increase in intracranial pressure

prednisolone $(1 \mathrm{mg} / \mathrm{kg} /$ day $)$ was also started $24 \mathrm{~h}$ after systemic acyclovir treatment.

After 3 days of the above treatment, slit-lamp examination revealed $3+$ cells in the anterior chamber, $2+$ cells in the anterior vitreous, and increased vitreous opacity (grade 2) in the right eye. On the fundus examination, the multiple whitish infiltrations were increased and newly developed vitreous and peripapillary hemorrhages were detected in the right eye (Fig. 3). At that time, the patient had symptoms of headache. T2-weighted MRIs of the brain performed to find the cause of the atypical ARN demonstrated a sub-acute or old hemorrhagic infarction in the right occipital lobe, and contrast-enhancing lesions in the right basal ganglia (Fig. 4a and b). The spinal tapping was performed in the department of neurology in our hospital at the time when the patient complained of headache, and intracranial pressure was $31 \mathrm{mmHg}$. This suggests that the possibility of Terson's syndrome due to suddenly increased intracranial pressure in this case [15].

In view of the patient's clinical features, immune status, and brain images, he was diagnosed as having VZV-mediated ARN with Terson's syndrome. On day 5 of the admission, the patient's visual acuity was reduced to counting fingers at $50 \mathrm{~cm}$ in the right eye. Retinal infiltration and vitreous hemorrhage were increased. Therefore, we started intravitreal
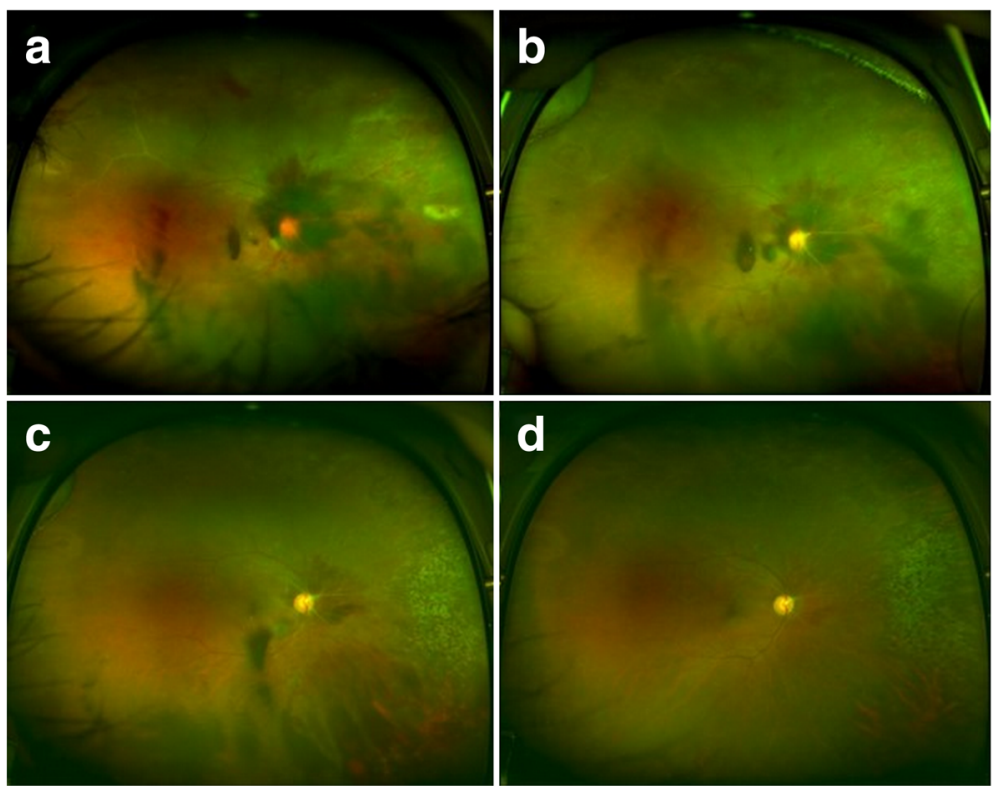

Fig. 5 Regression of atypical retinal necrosis in the right eye (a, b, c, d). a On day 11, the whitish retinal lesion was decreased. $\mathbf{b}, \mathbf{c}$ At 1 and 3 months after the initial visit, the peripheral retinal necrosis and vitreous hemorrhage have decreased. $\mathbf{d}$ Eight months after the first visit, the vitreous and peripapillary hemorrhage are nearly absorbed. Peripheral retinal necrosis is not detected 
injections of ganciclovir $(2 \mathrm{mg} / 0.5 \mathrm{~mL})$. After 5 intravitreal injections (from the 5 to 15 day of the admission), the multiple white-yellowish infiltrative lesions and retinal dot hemorrhages were decreased, and visual acuity increased to 20/800 in the right eye. The vitreous and peripapillary hemorrhages were also markedly decreased compared with the initial status of the ARN. After 20 days of intravenous antiviral therapy, the patient was discharged on oral valacyclovir, $1000 \mathrm{mg}$ twice a day, combined with an oral prednisolone (tapering dose, $0.5 \mathrm{~m} / \mathrm{kg} /$ day). After 5 intravitreal ganciclovir injections over a period of 8 months, there was no recurrence in the patient's right eye, in which his visual acuity had improved to 20/60 (Fig. 5a and b). The contralateral eye remained normal.

\section{Discussion and conclusions}

ARN is a rare vision-threatening disease characterized by rapidly progressive, peripheral retinal necrosis combined with inflammation of the vitreous or anterior chamber. Traditional treatment of ARN is induction with intravenous acyclovir followed by oral acyclovir. [12]. Here we have described a case of ARN with atypical clinical features that progressed to retinal necrosis accompanied by vitreous and peripapillary hemorrhage. Despite of aggressive treatment with systemic antiviral agent and steroids, the retinal necrosis progressed and a sub-acute or old hemorrhagic infarction was detected on MRI of the brain. The spinal tapping was performed in the department of neurology in our hospital at the time when the patient complained of headache, and intracranial pressure was $31 \mathrm{mmHg}$. The patient was diagnosed with VZV-mediated ARN combined with Terson's syndrome. The multiple whitish, infiltrating retinal lesions combined with peripapillary hemorrhage responded well to intravitreal ganciclovir injections. To our knowledge, there are no case reports on atypical ARN accompanied by Terson's syndrome. We also found that in the event of a poor response to traditional treatment such as intravenous acyclovir, intravitreal ganciclovir may have a role as an adjunctive therapy in patients of VZV associated ARN combined with Terson's syndrome.

\section{Abbreviations}

ANR: Acute retinal necrosis; HSV: Herpes simplex virus; IgG: Immunoglobulin G; MRI: Brain magnetic resonance imaging; PCR: Polymerase chain reaction; VZV: Varicella zoster virus

\section{Acknowledgements}

None.

\section{Funding}

No funding was obtained for this study.
Availability of data and materials

All the data supporting the conclusions of this article is included in the present article.

\section{Authors' contributions}

$J Y L$ was responsible for obtaining consent, collecting data and drafting the manuscript. DYK, HJL, JHJ, SPP, JYK has designed the overall concept, and revised the draft. All authors approved the final version.

\section{Ethics approval and consent to participate}

The local ethics committee ruled that no formal ethics approval was required in this case report.

The authors declare that they adhered to the CARE guidelines/methodology.

\section{Consent for publication}

Written informed consent was obtained from the patient for publication of this case report and any accompanying images.

\section{Competing interests}

The authors declare that they have no competing interests.

\section{Publisher's Note}

Springer Nature remains neutral with regard to jurisdictional claims in published maps and institutional affiliations.

\section{Author details}

'Department of Ophthalmology, Jeju National University Hospital, Jeju National University School of Medicine, 1753-3 Ara-1 Dong, Jeju-Si, Jeju Self-Governing Province, Republic of Korea. ${ }^{2}$ Department of Ophthalmology, Chungbuk National University College of Medicine, Cheongju, South Korea. ${ }^{3}$ Department of Ophthalmology, Kangdong Sacred Heart Hospital, Hallym University College of Medicine, Seoul, South Korea.

Received: 2 June 2017 Accepted: 11 December 2017

Published online: 19 December 2017

\section{References}

1. Lau CH, Missotten T, Salzmann J, Lightman SL. Acute retinal necrosis features, management, and outcomes. Ophthalmology. 2007;114:756-62.

2. Şimşek M, Özdal PÇ, Tekin K. Efficacy of oral Valacyclovir treatment in a case with acute retinal necrosis. Turk J Ophthalmol. 2017:47(3):174-9.

3. Young NJ, Bird AC. Bilateral acute retinal necrosis. Br J Ophthalmol. 1978;62: $581-90$

4. Culbertson WW, Blumenkranz MS, Pepose JS, Stewart JA, Curtin VT. Varicella zoster virus is a cause of the acute retinal necrosis syndrome. Ophthalmology. 1986;93:559-69.

5. Lewis ML, Culbertson WW, Post JD, Miller D, Kokame GT, Dix RD. Herpes simplex virus type 1: a cause of the acute retinal necrosis syndrome. Ophthalmology. 1989;96:875-8.

6. Walters G, James TE. Viral causes of the acute retinal necrosis syndrome. Curr Opin Ophthalmol. 2001;12:191-5.

7. Holland GN. Standard diagnosis criteria for the acute retinal necrosis syndrome. Executive Committee of the American Uveitis Society. Am J Ophthalmol. 1994;117:663-7.

8. De Boer JH, Luyendijk L, Rothova A, Baarsma GS, de Jong PT, Bollemeijer JG, et al. Detection of intraocular antibody production to herpesviruses in acute retinal necrosis syndrome. Am J Ophthalmol. 1994;117:201-10.

9. Fox GM, Crouse CA, Chuang EL, Pflugfelder SC, Cleary TJ, Nelson SJ, et al. Detection of herpesvirus DNA in vitreous and aqueous specimens by the polymerase chain reaction. Arch Ophthalmol. 1991;109:266-71.

10. Adleberg $\mathrm{JM}$, Wittwer $C$. Use of the polymerase chain reaction in the diagnosis of ocular disease. Curr Opin Ophthalmol. 1995;6:80-5.

11. Sugita S, Iwanaga $Y$, Kawaguchi $T$, et al. Detection of herpesvirus genome by multiplex polymerase chain reaction (PCR) and real-time PCR in ocular fluids of patients with acute retinal necrosis. Nippon Ganka Gakkai Zasshi. 2008;112:30-8.

12. Chau Tran TH, Cassoux N, Bodaghi B, Lehoang P. Successful treatment with combination of systemic antiviral drugs and intravitreal ganciclovir injections in the management of severe necrotizing herpetic retinitis. Ocul Immunol Inflamm. 2003;11(2):141-4. 
13. Wong R, Pavesio CE, Laidlaw DA, Williamson TH, Graham EM, Stanford MR. Acute retinal necrosis: the effects of intravitreal foscarnet and virus type on outcome. Ophthalmology. 2010;117(3):556-60.

14. Jabs DA, Nussenblatt RB, Rosenbaum JT. Standardization of Uveitis nomenclature (SUN) working group. Standardization of uveitis nomenclature for reporting clinical data.Results of the first international workshop. Am J Ophthalmol. 2005;140:509-16.

15. Vanderlinden RG, Chisholm LD. Vitreous hemorrhages and sudden increased intracranial pressure. J Neurosurg. 1974;41:167-76.

Submit your next manuscript to BioMed Central and we will help you at every step:

- We accept pre-submission inquiries

- Our selector tool helps you to find the most relevant journal

- We provide round the clock customer support

- Convenient online submission

- Thorough peer review

- Inclusion in PubMed and all major indexing services

- Maximum visibility for your research

Submit your manuscript at www.biomedcentral.com/submit
Biomed Central 